\title{
Predicting the dielectric nonlinearity of anisotropic composite materials via tensorial analysis
}

\author{
S Giordano ${ }^{1,3}$ and W Rocchia ${ }^{2}$ \\ ${ }^{1}$ Department of Physics, University of Cagliari, Cittadella Universitaria, I-09042 Monserrato, Italy \\ ${ }^{2}$ NEST CNR-INFM, Scuola Normale Superiore, Piazza dei Cavalieri 7, I-56126 Pisa, Italy \\ E-mail: stefano.giordano@dsf.unica.it andw.rocchia@sns.it
}

Received 13 September 2006, in final form 11 October 2006

Published 8 November 2006

Online at stacks.iop.org/JPhysCM/18/10585

\begin{abstract}
The discovery of new materials with peculiar optical properties as well as the prediction of their behaviour given the microstructure is a matter of remarkable interest in the community of material scientists. A complete theory allowing such a prediction is not yet available. We have formulated a theory able to analytically predict the effective second- and third-order nonlinear electrical behaviour of a dilute dispersion of randomly oriented anisotropic nonlinear spheres in a linear host. The inclusion medium has non-vanishing second- and third-order nonlinear hypersusceptibilities. As a result, the overall composite material is nonlinear but isotropic because of the random orientation of the inclusions. We derive the expressions for the equivalent permittivity and for the Kerr equivalent hypersusceptibility in terms of the characteristic electric tensors describing the electrical behaviour of the spheres. The complete averaging over inclusion positions and orientations led to general results in the dilute limit. We show that these results are consistent with earlier theories and that they provide null second-order hypersusceptibility as expected in a macroscopically isotropic medium. This theory generalizes the well-known Maxwell-Garnett formula and it can be easily specialized to any of the 32 crystallographic symmetry classes. Despite this study assuming static conditions, it can be generalized to the sinusoidal regime, pointing at an interesting way to engineer optically active materials with desired behaviour.
\end{abstract}

(Some figures in this article are in colour only in the electronic version)

\section{Introduction}

In recent years there has been considerable interest in the nonlinear response of anisotropic composite materials. This interest is due to several reasons. First, the anisotropic nonlinear

3 Author to whom any correspondence should be addressed. 
composites are important from the technological point of view, playing a central role in the study of the electrical breakdown and nonlinear optical phenomena. In particular, the enhanced nonlinear optical response in composites can be used to design novel optical devices. Furthermore, there is a strong theoretical interest in composite materials aimed to bring the applicability of the Maxwell [1], Maxwell-Garnett [2] and Bruggeman [3] approaches from linear approximations to more detailed ones.

The Maxwell-Garnett approximation [2] (also known as the Clausius-Mossotti approximation [4]) is one of the most widely used methods for calculating the equivalent dielectric properties of linear inhomogeneous materials. It describes a scenario where one phase is considered as a host in which inclusions of the other phase are embedded. It involves an exact evaluation of the uniform electric field induced inside a single spherical [1], or ellipsoidal [5, 6], inclusion and the calculation of the electrical observables averaged over the mixture volume. The dilute limit is assumed, so that each inclusion basically 'feels' only the uniform, externally applied, electric field and behaves as an electric dipole. This approach has been extensively used for studying the properties of two-component mixtures in which both the host and the inclusions are isotropic. Moreover, a lot of work has been devoted to describe the relationship between microstructure and electrical properties. A fundamental result is given by the HashinShtrikman variational analysis [7], which provides upper and lower bounds for composite materials, irrespective of their microstructure. A method of finding the relationship between the spatial correlation function of the dispersed medium and the final properties of the material is derived from the Brown expansion [8].

Many attempts have been made in order to take into account dielectric nonlinearity in the constitutive equations of either phase. A systematic perturbation expansion method was developed and employed to solve electrostatic and quasi-static boundary value problems of weakly nonlinear media [9]. The results have been verified by means of numerical simulation [10].

A lot of work has also been devoted to the analysis of the huge enhancing, caused by local field effects, of the effective cubic nonlinearity in random structures of polycrystals [11-16]. A similar nonlinear electrical behaviour has been found in dispersions of randomly oriented dielectrically nonlinear ellipsoids [17].

From the point of view of anisotropy, the effective dielectric function of a medium of anisotropic inclusions embedded in an isotropic host has been calculated using several generalizations of the Maxwell-Garnett approximation [18-21].

Finally, the problems of second and third harmonic generation in random, dielectrically nonlinear composites have been tackled and fully general expressions for the equivalent secondand third-order hypersusceptibilities have been given in terms of the nonlinear behaviour of the constituents $[22,23]$; however, these expressions cannot be evaluated exactly for most random composites.

In this paper, we present a variation of the Maxwell-Garnett approach that provides explicit, analytical results applicable to mixtures where the host is a linear isotropic material and the randomly dispersed inclusions are made of nonlinear anisotropic spheres. Here, and throughout the paper, when mentioning nonlinearity, we mean a nonlinear relationship between electric field and electric displacement. The random orientation of all the spherical grains results in an overall nonlinear but still isotropic behaviour of the composite. We express the dielectric behaviour up to the third-order hypersusceptibility in terms of the electrical constitutive tensors of the grains. Our method is based on the exact field calculation inside a single inclusion, followed by an averaging process, and it only assumes the dilute limit.

For many practical applications one may need to design composite materials with desired electro-optic behaviour. In this work, we make available some analytical results of general 
applicability, obtained by modelling the dielectric properties of a complex distribution of particles.

\section{Electric field inside a single, fixed, nonlinear and anisotropic sphere}

Let us first introduce anisotropy; a linear anisotropic sphere is characterized by its constant permittivity tensor $\varepsilon$ (constitutive equation $\mathbf{D}_{s}=\varepsilon \mathbf{E}_{s}$, where $\mathbf{E}_{s}$ and $\mathbf{D}_{s}$ are electric field and displacement, respectively, inside the inclusion). The sphere is embedded in an isotropic medium with scalar permittivity $\varepsilon_{0}$ and it is subjected to a uniform electric field $\mathbf{E}_{0}$. The electric field inside the sphere, due to the application of $\mathbf{E}_{0}$, is uniform, and is given by [24, 25]

$$
\mathbf{E}_{s}=3 \varepsilon_{0}\left[\varepsilon+2 \varepsilon_{0} I\right]^{-1} \mathbf{E}_{0} .
$$

Equation (1), where $I$ is the identity tensor, represents a generalization of the Lorentz field formula for anisotropic linear spheres. As expected, the internal electric field may very well be not aligned with $\mathbf{E}_{0}$. The relationship (1) can be extended to the case of nonlinear media described by the constitutive equation $\mathbf{D}_{s}=\varepsilon\left(\mathbf{E}_{s}\right) \mathbf{E}_{s}$, where the permittivity tensor $\varepsilon\left(\mathbf{E}_{s}\right)$ depends arbitrarily on the internal electric field $\mathbf{E}_{s}$ :

$$
\mathbf{E}_{s}=3 \varepsilon_{0}\left[\varepsilon\left(\mathbf{E}_{s}\right)+2 \varepsilon_{0} I\right]^{-1} \mathbf{E}_{0} .
$$

Actually, equation (2) implicitly determines the unknown electric field $\mathbf{E}_{s}$. This is true since the electric field fulfilling equation (2) satisfies both Maxwell's laws and the boundary conditions at the inclusion surface as its linear counterpart (1) does when $\varepsilon=\varepsilon\left(\mathbf{E}_{s}\right)$. Equation (2) can be seen as the generalization of the Lorentz field formula to anisotropic and nonlinear spheres. This very simple property has several interesting consequences. It is worth saying that any kind of anisotropy can be modelled through the procedure here presented, including uniaxial, biaxial and also gyrotropic media, in which the permittivity tensor contains an antisymmetric part. However, in the following, we consider a symmetric permittivity tensor in order to exploit diagonalization by means of a suitable orthogonal matrix; this is done for the sake of simplicity in the exposition and it does not restrict the generality of the presented approach.

Let us consider the following expansion of the permittivity tensor:

$$
\varepsilon_{i j}\left(\mathbf{E}_{s}\right)=\varepsilon_{i j}^{0}+\eta_{i j k} E_{k}+\chi_{i j k h} E_{k} E_{h} .
$$

Here $E_{k}$ represents the $k$ th component of $\mathbf{E}_{s}$ in an arbitrary basis. Throughout the paper we adopt the Einstein (repeated subscripts in products) summation convention unless the explicit summation symbol is used. The tensors appearing in equation (3) have some well-known symmetry rules, which descend from the existence of a thermodynamic potential (the Gibbs free energy) for the crystal: the first-order linear permittivity tensor $\varepsilon_{i j}^{0}$ is symmetric in the subscripts $i$ and $j$. The second-order hypersusceptibility tensor $\eta_{i j k}$ is symmetric in the subscripts $j$ and $k$ (18 independent entries). The third-order hypersusceptibility tensor $\chi_{i j k h}$ is invariant on any permutation of the three last subscripts $j, k$ and $h$ (30 independent entries) $[25,26]$. These properties, named internal symmetries, can be synthesized as follows:

$$
\begin{aligned}
& \varepsilon_{i j}^{0}=\varepsilon_{j i}^{0}, \quad \eta_{i j k}=\eta_{i k j}, \\
& \chi_{i j k h}=\chi_{i j h k}=\chi_{i k j h}=\chi_{i h j k}=\chi_{i k h j}=\chi_{i h k j} .
\end{aligned}
$$

Other symmetry rules are fulfilled when the tensors refer to a material belonging to one of the 32 admissible crystal classes (see appendix A for details).

Moreover, appendix B contains the detailed solution of equation (2) in the basis that diagonalizes the permittivity tensor of a single sphere; it provides the explicit form for the 
electric field inside a nonlinear anisotropic sphere:

$$
\begin{gathered}
E_{i}=\frac{3 \varepsilon_{0}}{\varepsilon_{j}+2 \varepsilon_{0}} E_{0 j} \delta_{i j}-\frac{9 \varepsilon_{0}^{2} \eta_{i j k}}{\left(\varepsilon_{i}+2 \varepsilon_{0}\right)\left(\varepsilon_{j}+2 \varepsilon_{0}\right)\left(\varepsilon_{k}+2 \varepsilon_{0}\right)} E_{0 j} E_{0 k} \\
+\frac{\left(2 \frac{\eta_{i j q} \eta_{q k h}}{\varepsilon_{q}+2 \varepsilon_{0}}-\chi_{i j k h}\right) 27 \varepsilon_{0}^{3}}{\left(\varepsilon_{i}+2 \varepsilon_{0}\right)\left(\varepsilon_{j}+2 \varepsilon_{0}\right)\left(\varepsilon_{k}+2 \varepsilon_{0}\right)\left(\varepsilon_{h}+2 \varepsilon_{0}\right)} E_{0 j} E_{0 k} E_{0 h} .
\end{gathered}
$$

Here we have considered the diagonal linear permittivity $\varepsilon_{i j}^{0}=\varepsilon_{i} \delta_{i j}$.

Equation (5) is exact up to the third order in the external electric field and represents a strong generalization of the Lorentz field.

\section{Electric field inside a single, randomly oriented, nonlinear and anisotropic sphere}

So far, we have used a fixed coordinate system, corresponding to the principal directions of the permittivity tensor of the sphere. Now, we will consider an arbitrary reference frame in order to compute the average value of the internal electric field when the sphere is randomly oriented in the space. Let $\mathbf{n}_{1}, \mathbf{n}_{2}$ and $\mathbf{n}_{3}$ be unit vectors determining the principal directions of the sphere in space; the vectorial expression of the internal electric field is given by

$\mathbf{E}_{s}=\left\{a_{j} \delta_{i j}\left(\mathbf{E}_{0} \mathbf{n}_{j}\right)+b_{i j k}\left(\mathbf{E}_{0} \mathbf{n}_{j}\right)\left(\mathbf{E}_{0} \mathbf{n}_{k}\right)+c_{i j k h}\left(\mathbf{E}_{0} \mathbf{n}_{j}\right)\left(\mathbf{E}_{0} \mathbf{n}_{k}\right)\left(\mathbf{E}_{0} \mathbf{n}_{h}\right)\right\} \mathbf{n}_{i}$,

where the coefficients $a, b$ and $c$ have been defined in appendix B.

We now want to average $\mathbf{E}_{s}$ over all the possible orientations of the sphere in the space:

$\left\langle E_{r}\right\rangle=a_{j} \delta_{i j} E_{0 l}\left\langle n_{j l} n_{i r}\right\rangle+b_{i j k} E_{0 l} E_{0 s}\left\langle n_{j l} n_{k s} n_{i r}\right\rangle+c_{i j k h} E_{0 l} E_{0 s} E_{0 t}\left\langle n_{j l} n_{k s} n_{h t} n_{i r}\right\rangle$,

where $n_{j k}$ is the $k$ th component of the unit vector $\mathbf{n}_{j}$.

The detailed description of the averaging process is quite cumbersome and it is left to the reader in appendix C. In fact, equations (C.3), (C.4) and (C.10) can be used to simplify equation (7), yielding the value of the electric field inside the ellipsoid averaged over all its possible orientations:

$$
\left\langle\mathbf{E}_{s}\right\rangle=\alpha \mathbf{E}_{0}+\beta E_{0}^{2} \mathbf{E}_{0},
$$

where we have defined the following averaging parameters, and made explicit every summation process:

$\alpha=\sum_{i} \frac{\varepsilon_{0}}{\varepsilon_{i}+2 \varepsilon_{0}}$

$\beta=\sum_{i, k} \frac{9 \varepsilon_{0}^{3}}{5\left(\varepsilon_{i}+2 \varepsilon_{0}\right)^{2}\left(\varepsilon_{k}+2 \varepsilon_{0}\right)^{2}}\left(2 \sum_{q} \frac{\eta_{i i q} \eta_{q k k}}{\varepsilon_{q}+2 \varepsilon_{0}}-3 \chi_{i i k k}+4 \sum_{q} \frac{\eta_{i k q} \eta_{q i k}}{\varepsilon_{q}+2 \varepsilon_{0}}\right)$.

It is important to observe that the second-order term vanishes; this is coherent with the random orientation hypothesis. From the mathematical point of view, this fact is well explained by the presence of the skew-symmetric tensor defined in equation (C.4). When it is multiplied by symmetric tensors (see equations (7) and (B.4)), the result is zero.

\section{Dispersion of spheres}

If we now consider a mixture with a volume fraction $c \ll 1$ of randomly oriented, dielectrically anisotropic and nonlinear spheres embedded in a homogeneous matrix with permittivity $\varepsilon_{0}$, we can evaluate a different kind of average, the space average of the electric field over all of the volume occupied by the mixture. This can be done via the following relationship:

$$
\langle\mathbf{E}\rangle=c\left\langle\mathbf{E}_{s}\right\rangle+(1-c) \mathbf{E}_{0} .
$$


In the present approximation we do not take into account the interactions among the inclusions because of the very low concentration; therefore, each sphere behaves as an isolated one, subjected to the externally applied uniform electric field $\mathbf{E}_{0}$.

To evaluate the equivalent constitutive equation, we compute the average value of the displacement vector $\mathbf{D}(\mathbf{r})$ inside the random material. The symbol $V$ indicates the total space region occupied by the mixture, $V_{\mathrm{e}}$ the region occupied by the inclusions, and $V_{\mathrm{o}}$ the remaining space (so that $V=V_{\mathrm{e}} \cup V_{\mathrm{o}}$ ). The average value of $\mathbf{D}(\mathbf{r})$ is evaluated as follows ( $\mathbf{D}$ and $\mathbf{E}$ represent the local fields, $\langle\mathbf{D}\rangle$ and $\langle\mathbf{E}\rangle$ their macroscopic counterparts):

$$
\begin{aligned}
\langle\mathbf{D}\rangle=\frac{1}{|V|} \int_{V} \mathbf{D}(\mathbf{r}) \mathrm{d} \mathbf{r}=\frac{1}{|V|} \varepsilon_{0} \int_{V_{\mathrm{o}}} \mathbf{E}(\mathbf{r}) \mathrm{d} \mathbf{r}+\frac{1}{|V|} \int_{V_{\mathrm{e}}} \varepsilon(\mathbf{E}) \mathbf{E}(\mathbf{r}) \mathrm{d} \mathbf{r} \\
=\frac{1}{|V|} \varepsilon_{0} \int_{V_{\mathrm{o}}} \mathbf{E}(\mathbf{r}) \mathrm{d} \mathbf{r}+\frac{1}{|V|} \varepsilon_{0} \int_{V_{\mathrm{e}}} \mathbf{E}(\mathbf{r}) \mathrm{d} \mathbf{r}+\frac{1}{|V|} \int_{V_{\mathrm{e}}} \varepsilon(\mathbf{E}) \mathbf{E}(\mathbf{r}) \mathrm{d} \vec{r} \\
\quad-\frac{1}{|V|} \varepsilon_{0} \int_{V_{\mathrm{e}}} \mathbf{E}(\mathbf{r}) \mathrm{d} \mathbf{r}=\varepsilon_{0}\langle\mathbf{E}\rangle+c\left\langle\mathbf{D}_{s}\right\rangle-c \varepsilon_{0}\left\langle\mathbf{E}_{s}\right\rangle .
\end{aligned}
$$

The average value of $\left\langle\mathbf{D}_{s}\right\rangle$ can be obtained by observing that $\mathbf{D}_{s}+2 \varepsilon_{0} \mathbf{E}_{s}=3 \varepsilon_{0} \mathbf{E}_{0}$ (see equation (2)) and therefore

$$
\left\langle\mathbf{D}_{s}\right\rangle=\varepsilon_{0}\left(3 \mathbf{E}_{0}-2\left\langle\mathbf{E}_{s}\right\rangle\right) \text {. }
$$

So, we obtain from equations (11) and (12)

$$
\langle\mathbf{D}\rangle=\varepsilon_{0}\langle\mathbf{E}\rangle+3 c \varepsilon_{0} \mathbf{E}_{0}-3 c \varepsilon_{0}\left\langle\mathbf{E}_{s}\right\rangle .
$$

From equations (8), (10), and (13) it follows that all the averaged vectorial quantities are aligned with $\mathbf{E}_{0}$; therefore, we can continue our computations with scalar quantities; moreover, from now on, we will leave out the average symbols $\langle\cdot\rangle$. Equations (8), (10), and (13) may then be rewritten as

$$
\begin{aligned}
& E_{s}=\alpha E_{0}+\beta E_{0}^{3} \\
& E=c E_{s}+(1-c) E_{0} \\
& D=\varepsilon_{0} E+3 c \varepsilon_{0} E_{0}-3 c \varepsilon_{0} E_{s} .
\end{aligned}
$$

These are the main equations describing the overall mixture behaviour. We shall recall the approximations introduced to obtain them: the first equation is exact from the volume fraction point of view, but it is approximated from the nonlinearity point of view; in fact, it accounts for just the first nonlinear terms (up to the third order). The second equation, dealing with the average value of the electric field, has been deduced under the hypothesis of low concentration. The third one is exact. By solving system (19), we search for a relation between $D$ and $E$ characterizing the nonlinear mixture. By eliminating $E_{s}$ from the first two relationships, we obtain

$$
E=(1-c+c \alpha) E_{0}+c \beta E_{0}^{3} .
$$

We now need to solve the previous equation with respect to $E_{0}$ : for our purposes it is sufficient to obtain the first terms of a series solution and thus we let $E_{0}=\lambda E+\mu E^{3}$, we substitute it in equation (15) and we solve for the unknown coefficients; the result is

$$
E_{0}=\frac{E}{1-c+c \alpha}-\frac{c \beta E^{3}}{(1-c+c \alpha)^{4}} .
$$

The final result is obtained, after a very long but straightforward calculation, by substituting equation (16) in the third equation of system (14):

$$
D=\varepsilon_{0} \frac{1+2 c-2 c \alpha}{1-c+c \alpha} E-\frac{3 c \beta \varepsilon_{0} E^{3}}{(1-c+c \alpha)^{4}} .
$$


As we will show later on, this expression is a generalization of the standard Maxwell mixing formula for linear isotropic spheres in linear isotropic matrix. It can be written in the simple isotropic form $D=\varepsilon_{\mathrm{eq}} E+\chi_{\mathrm{eq}} E^{3}$, where

$$
\begin{gathered}
\varepsilon_{\mathrm{eq}}=\varepsilon_{0} \frac{1+2 c-2 c \alpha}{1-c+c \alpha}=\varepsilon_{0} \frac{1+2 c-2 c \sum_{i} \frac{\varepsilon_{0}}{\varepsilon_{i}+2 \varepsilon_{0}}}{1-c+c \sum_{i} \frac{\varepsilon_{0}}{\varepsilon_{i}+2 \varepsilon_{0}}} \\
\chi_{\mathrm{eq}}=-\frac{3 c \beta \varepsilon_{0}}{(1-c+c \alpha)^{4}}=-\frac{27 c \varepsilon_{0}^{4}}{\left(1-c+c \sum_{j} \frac{\varepsilon_{0}}{\varepsilon_{j}+2 \varepsilon_{0}}\right)^{4}} \\
\times \sum_{i, k} \frac{\left(2 \sum_{q} \frac{\eta_{i i q} \eta_{q k k}}{\varepsilon_{q}+2 \varepsilon_{0}}-3 \chi_{i i k k}+4 \sum_{q} \frac{\eta_{i k q} \eta_{q i k}}{\varepsilon_{q}+2 \varepsilon_{0}}\right)}{5\left(\varepsilon_{i}+2 \varepsilon_{0}\right)^{2}\left(\varepsilon_{k}+2 \varepsilon_{0}\right)^{2}} .
\end{gathered}
$$

Summarizing, we have expressed the equivalent linear permittivity and the equivalent Kerr third-order hypersusceptibility in terms of the parameters of the composite material: the volume fraction $c$, the host linear permittivity $\varepsilon_{0}$ and the characteristic tensors $\varepsilon, \eta$ and $\chi$.

The expression for the linear equivalent permittivity can be written in a very simple form by letting $s=\sum_{j}\left(\varepsilon_{j}-\varepsilon_{0}\right) /\left(\varepsilon_{j}+2 \varepsilon_{0}\right)$. In fact, the first formula in equation (18) can be recast in the following form, where the first-order expansion in the volume fraction $c$ is also shown:

$$
\varepsilon_{\mathrm{eq}}=\varepsilon_{0}\left[1+\frac{3 c s}{3-c s}\right] \cong \varepsilon_{0}[1+c s] .
$$

The linear and nonlinear macroscopic quantities describing the isotropic mixture must be invariant with respect to the framework adopted. In appendix D we show that the equivalent permittivity $\varepsilon_{\mathrm{eq}}$ and the equivalent hypersusceptibility $\chi_{\mathrm{eq}}$ are two actual invariants of the system.

\section{Consistency with earlier theories: isotropic spheres}

To test the theory, we applied it to the simpler case where spheres are isotropic. The isotropy of the spheres leads to the following conditions on the constitutive tensors:

$$
\begin{aligned}
& \varepsilon_{i j}=\varepsilon_{s} \delta_{i j} \quad \text { or } \quad \varepsilon_{1}=\varepsilon_{2}=\varepsilon_{3}=\varepsilon_{s} \\
& \eta_{i j k}=0 \quad \forall i, j, k \\
& \chi_{i j k h}=\frac{1}{3} \chi_{s}\left(\delta_{i j} \delta_{k h}+\delta_{i k} \delta_{j h}+\delta_{i h} \delta_{j k}\right) .
\end{aligned}
$$

Since the medium is isotropic, it is described by only two parameters, namely $\varepsilon_{s}$ and $\chi_{s}$, the tensor $\eta$ being null. Moreover, one can observe that the form of the tensor $\chi$ is the symmetrized version of the asymmetric tensor $\tilde{\chi}$ defined as $\tilde{\chi}_{i j k h}=\chi_{s} \delta_{i j} \delta_{k h}$ (see appendix $\mathrm{D}$ for the description of a symmetrization process). In such a case, the application of equations (17) and (18) gives the following result:

$$
D(E)=\overbrace{\varepsilon_{0} \frac{2 \varepsilon_{0}+\varepsilon_{s}-2 c\left(\varepsilon_{0}-\varepsilon_{s}\right)}{2 \varepsilon_{0}+\varepsilon_{s}+c\left(\varepsilon_{0}-\varepsilon_{s}\right)}}^{\text {Maxwell-Garnett }} E+\frac{81 c \varepsilon_{0}^{4}}{\left[2 \varepsilon_{0}+\varepsilon_{s}+c\left(\varepsilon_{0}-\varepsilon_{s}\right)\right]^{4}} \chi_{s} E^{3} .
$$

This result, whose first term can be recognized as the standard Maxwell-Garnett formula, is well known in the literature concerning nonlinear isotropic composite materials and it shows the coherence of this theory with the earlier ones [17]. 
Table 1. List of the 32 crystallographic symmetry classes. For each class, the number of independent entries for tensors in spaces $\left[\mathrm{V}^{2}\right], \mathrm{V}\left[\mathrm{V}^{2}\right]$ and $\mathrm{V}\left[\mathrm{V}^{3}\right]$ are reported, adopting the system of notation introduced by Jahn [27] and Sirotine [28].

\begin{tabular}{|c|c|c|c|c|c|}
\hline Crystal system & Crystal class & Name of class & {$\left[\mathrm{V}^{2}\right]$} & $\mathrm{V}\left[\mathrm{V}^{2}\right]$ & $\mathrm{V}\left[\mathrm{V}^{3}\right]$ \\
\hline \multirow{3}{*}{ Triclinic } & 1 & Pedial & 6 & 18 & 30 \\
\hline & $\overline{1}$ & Pinacoidal & 6 & - & 30 \\
\hline & 2 & Sphenoidal & 4 & 8 & 16 \\
\hline \multirow[t]{3}{*}{ Monoclinic } & $m$ & Domatic & 4 & 10 & 16 \\
\hline & $2 / m$ & Prismatic & 4 & - & 16 \\
\hline & 222 & Rhombic-disphenoidal & 3 & 3 & 9 \\
\hline \multirow[t]{5}{*}{ Orthorhombic } & $m m 2(2 m m)$ & Rhombic-pyramidal & 3 & 5 & 9 \\
\hline & $2 / m 2 / m 2 / m$ & Rhombic-dipyramidal & 3 & - & 9 \\
\hline & 4 & Tetragonal-pyramidal & 2 & 4 & 8 \\
\hline & $\overline{4}$ & Tetragonal-disphenoidal & 2 & 4 & 8 \\
\hline & $4 / m$ & Tetragonal-dipyramidal & 2 & - & 8 \\
\hline \multirow[t]{9}{*}{ Tetragonal } & 422 & Tetragonal-trapezohedral & 2 & 1 & 5 \\
\hline & $4 m m$ & Ditetragonal-pyramidal & 2 & 3 & 5 \\
\hline & $\overline{4} 2 m$ & Tetragonal-scalenohedral & 2 & 2 & 5 \\
\hline & $4 / m 2 / m 2 / m$ & Ditetragonal-dipyramidal & 2 & - & 5 \\
\hline & 3 & Trigonal-pyramidal & 2 & 6 & 10 \\
\hline & $\overline{3}$ & Rhombohedral & 2 & - & 10 \\
\hline & 32 & Trigonal-trapezohedral & 2 & 2 & 6 \\
\hline & $3 \mathrm{~m}$ & Ditrigonal-pyramidal & 2 & 4 & 6 \\
\hline & $\overline{3} 2 / m$ & Hexagonal-scalenohedral & 2 & - & 6 \\
\hline \multirow[t]{9}{*}{ Hexagonal } & 6 & Hexagonal-pyramidal & 2 & 4 & 6 \\
\hline & $\overline{6}$ & Trigonal-dipyramidal & 2 & 2 & 6 \\
\hline & $6 / m$ & Hexagonal-dipyramidal & 2 & - & 6 \\
\hline & 622 & Hexagonal-trapezohedral & 2 & 1 & 4 \\
\hline & $6 m m$ & Dihexagonal-pyramidal & 2 & 3 & 4 \\
\hline & $\overline{6} m 2$ & Ditrigonal-dipyramidal & 2 & 1 & 4 \\
\hline & $6 / m 2 / m 2 / m$ & Dihexagonal-dipyramidal & 2 & - & 4 \\
\hline & 23 & Tetaroidal & 1 & 1 & 3 \\
\hline & $2 / m \overline{3}$ & Diploidal & 1 & - & 3 \\
\hline \multirow[t]{3}{*}{ Isometric } & 432 & Gyroidal & 1 & - & 2 \\
\hline & $\overline{4} 3 m$ & Hextetrahedral & 1 & 1 & 2 \\
\hline & $4 / m \overline{3} 2 / m$ & Hexoctahedral & 1 & - & 2 \\
\hline
\end{tabular}

\section{Examples of application}

The general solution given by equations (17) and (18) can be specialized to each crystal class. In fact, the general forms of tensors belonging to $\mathrm{V}\left[\mathrm{V}^{2}\right]$ or $\mathrm{V}\left[\mathrm{V}^{3}\right]$ (see appendix A for details and nomenclature) are known for every crystal class and depend only on a limited number of parameters, that we list in table 1 for the convenience of the reader. The detailed expressions for the tensors can be found in the literature [25]. We will show some applications of this procedure in the following.

\subsection{Hextetrahedral class}

This class contains, among other compounds, the following ones: sodium aluminium silicate chloride (sodalite), zinc iron sulfide (sphalerite), copper arsenide (domeykite) and sodium calcium aluminium silicate sulfur sulfate (lazurite). 
Let us consider spheres constituted by a crystal member of the hextetrahedral class $(\overline{4} 3 \mathrm{~m})$. For such a class one can find the following forms for the tensors [25]. The linear permittivity tensor for such crystals is isotropic and therefore we have

$$
\varepsilon_{i j}=\varepsilon_{s} \delta_{i j} \quad \text { or } \quad \varepsilon_{1}=\varepsilon_{2}=\varepsilon_{3}=\varepsilon_{s} .
$$

The second-order hypersusceptibility tensor is described by one parameter in the following way:

$$
\eta_{123}=\eta_{132}=\eta_{213}=\eta_{231}=\eta_{321}=\eta_{312}=\eta_{s}
$$

All the terms not mentioned in equation (23) are null. Finally, the third-order hypersusceptibility tensor is described by two parameters in the following way:

$$
\begin{aligned}
& \chi_{1111}=\chi_{2222}=\chi_{3333}=\chi_{s a} \\
& \chi_{1122}=\chi_{2233}=\chi_{3311}=\chi_{1133}=\chi_{2211}=\chi_{3322}=\frac{1}{3} \chi_{s b} .
\end{aligned}
$$

The other terms can be derived by the symmetry rules given in equation (4) or are null. The anisotropic nonlinear electrical behaviour of this kind of crystal is defined by the four parameters $\varepsilon_{s}, \eta_{s}, \chi_{s a}$ and $\chi_{s b}$. The direct application of equations (17) and (18) gives the result:

$$
D(E)=\overbrace{\varepsilon_{0} \frac{2 \varepsilon_{0}+\varepsilon_{s}-2 c\left(\varepsilon_{0}-\varepsilon_{s}\right)}{2 \varepsilon_{0}+\varepsilon_{s}+c\left(\varepsilon_{0}-\varepsilon_{s}\right)}}^{\text {Maxwell-Garnett }} E+\frac{81 c \varepsilon_{0}^{4}\left(3 \chi_{s a}+2 \chi_{s b}-\frac{8 \eta_{s}^{2}}{2 \varepsilon_{0}+\varepsilon_{s}}\right)}{5\left[2 \varepsilon_{0}+\varepsilon_{s}+c\left(\varepsilon_{0}-\varepsilon_{s}\right)\right]^{4}} E^{3} .
$$

If the parameters $\chi_{s a}$ and $\chi_{s b}$ assume the same value and $\eta_{s}=0$ we again find equation (21), which is correct for the isotropic case.

\subsection{Tetragonal-scalenohedral class}

As a second example, we take into consideration spheres constituted by crystals belonging to the tetragonal-scalenohedral class $(\overline{4} 2 \mathrm{~m})$. It contains, among other compounds, copper iron sulfide (chalcopyrite) and calcium zinc silicate (hardystonite). For such a class, one can find in the literature [25] the following forms of the constitutive tensors. The linear permittivity tensor for such crystals is uniaxial, and therefore we have

$$
\varepsilon_{1}=\varepsilon_{2}=\varepsilon_{s a}, \quad \varepsilon_{3}=\varepsilon_{s b} .
$$

The second-order hypersusceptibility tensor is described by two parameters in the following way:

$$
\eta_{123}=\eta_{132}=\eta_{213}=\eta_{231}=\eta_{s a}, \quad \eta_{321}=\eta_{312}=\eta_{s b} .
$$

Again, all the terms not appearing in the previous equation (27) are null. Finally, the third-order hypersusceptibility tensor is described by five parameters in the following way:

$\chi_{1111}=\chi_{2222}=\chi_{s a}, \quad \chi_{3333}=\chi_{s b}$,

$\chi_{1122}=\chi_{2211}=\frac{\chi_{s c}}{3}, \quad \chi_{1133}=\chi_{2233}=\frac{\chi_{s d}}{3}, \quad \chi_{3311}=\chi_{3322}=\frac{\chi_{s e}}{3}$.

The other terms can be derived by the symmetry rules given in equation (4) or are null. The straightforward application of equations (17) and (18) provides the result:

$$
\begin{aligned}
& D(E)=\varepsilon_{0} \frac{1+2 c-2 c \varepsilon_{0} \frac{\varepsilon_{s a}+2 \varepsilon_{s b}+6 \varepsilon_{0}}{\left(\varepsilon_{s a}+2 \varepsilon_{0}\right)\left(\varepsilon_{s b}+2 \varepsilon_{0}\right)}}{1-c+c \varepsilon_{0} \frac{\varepsilon_{s a}+2 \varepsilon_{s b}+6 \varepsilon_{0}}{\left(\varepsilon_{s a}+2 \varepsilon_{0}\right)\left(\varepsilon_{s b}+2 \varepsilon_{0}\right)}} E+\frac{27 c \varepsilon_{0}^{4}}{\left[1-c+c \varepsilon_{0} \frac{\varepsilon_{s a}+2 \varepsilon_{s b}+6 \varepsilon_{0}}{\left(\varepsilon_{s a}+2 \varepsilon_{0}\right)\left(\varepsilon_{s b}+2 \varepsilon_{0}\right)}\right]^{4}} \\
& \times\left\{\frac{3}{5}\left[2 \frac{\chi_{s a}+\frac{1}{3} \chi_{s c}}{\left(\varepsilon_{s a}+2 \varepsilon_{0}\right)^{4}}+\frac{2}{3} \frac{\chi_{s d}+\chi_{s e}}{\left(\varepsilon_{s a}+2 \varepsilon_{0}\right)^{2}\left(\varepsilon_{s b}+2 \varepsilon_{0}\right)^{2}}+\frac{\chi_{s b}}{\left(\varepsilon_{s b}+2 \varepsilon_{0}\right)^{4}}\right]\right. \\
& \left.-\frac{8 \eta_{s a}}{5\left(\varepsilon_{s b}+2 \varepsilon_{0}\right)\left(\varepsilon_{s a}+2 \varepsilon_{0}\right)^{3}}\left[\frac{\eta_{s b}}{\left(\varepsilon_{s a}+2 \varepsilon_{0}\right)}+\frac{\eta_{s a}+\eta_{s b}}{\left(\varepsilon_{s b}+2 \varepsilon_{0}\right)}\right]\right\} E^{3} \text {. }
\end{aligned}
$$




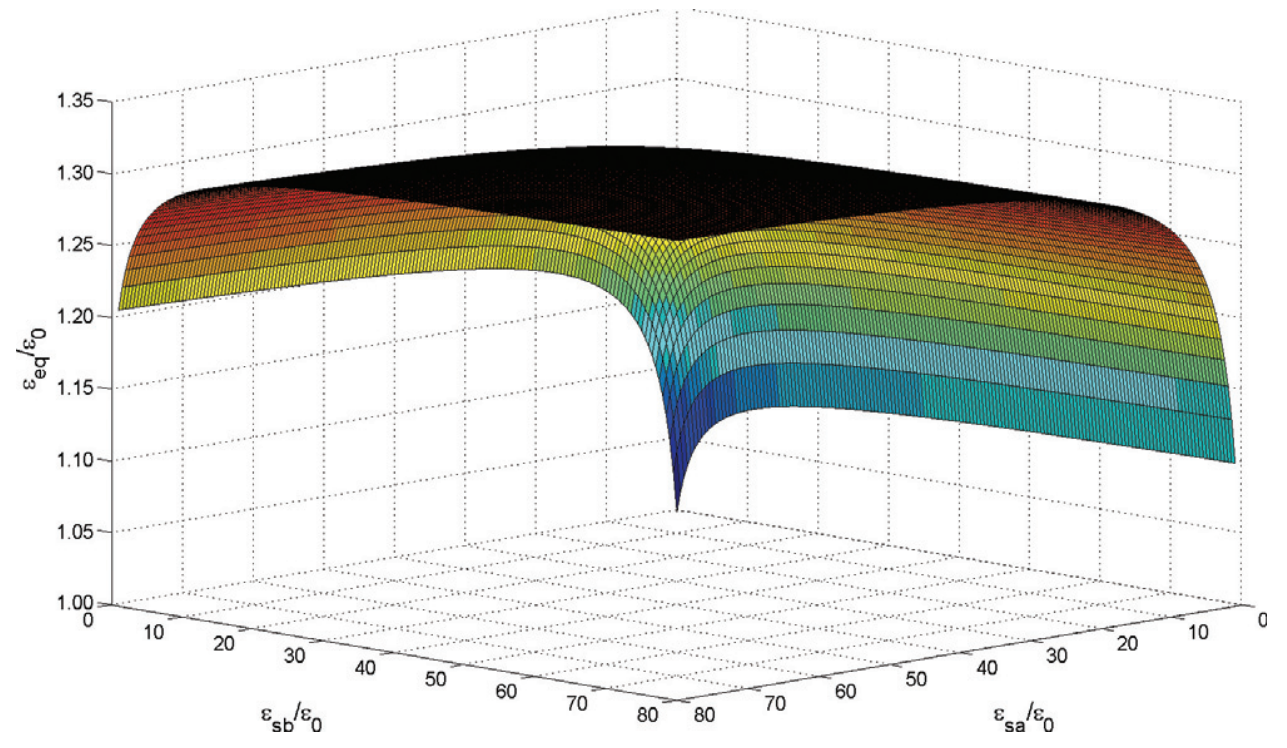

Figure 1. Composite equivalent permittivity relative to matrix permittivity when inclusions belong to the tetragonal-scalenohedral crystal class. The dependence is considered versus the two parameters describing the uniaxial permittivity tensor of the spherical inclusions, namely $\varepsilon_{s a}$ and $\varepsilon_{s b}$; in this chart, a fractional volume value of $c=0.1$ is used.

In figure 1, we show the dependence of the equivalent linear permittivity on the parameters that characterize the permittivity tensor of the inclusions. Due to its homogenous nature, we were able to present a chart which is independent on the permittivity of the hosting medium.

This chart indicates that the equivalent permittivity is upper bounded: the bound is approached as the inclusions increase their polarizability; its theoretical value can be calculated, yielding the value of $(1+2 c) /(1-c)$. In figure 2 , the influence of inclusion permittivity over the third-order susceptibility is studied.

Interestingly, this chart suggests that the third-order effects are enhanced when the linear permittivity of the inclusions is close to that of the matrix.

In figure 3, we show the effects of inclusion second- and third-order susceptibilities. In order to capture the general behaviour without getting lost in the details given by the large number of parameters, we made a specific assignment that allows considering when the inclusion second-order term prevails over the third-order term and vice versa. The chart shows the expected linear increasing dependence of $\chi_{\mathrm{eq}}$ on $\chi_{\mathrm{s} b}$.

A rough analysis shows that the electric field values for which the nonlinear effects show up are between $1 \times 10^{8}$ and $1 \times 10^{9}\left(\mathrm{~V} \mathrm{~m}^{-1}\right)$.

As a consistency check, we see that if $\varepsilon_{s a}=\varepsilon_{s b}, \eta_{s a}=\eta_{s b}, \chi_{s a}=\chi_{s b}$ and $\chi_{s c}=\chi_{s d}=$ $\chi_{s e}$ the tetragonal-scalenohedral class $(\overline{4} 2 \mathrm{~m})$ reduces to the hextetrahedral class $(\overline{4} 3 \mathrm{~m})$ and thus equation (29) becomes identical to equation (25).

For other crystal classes the number of independent elements in the constitutive electrical tensors is much higher and the results, very complicated from the algebraic point of view, are not reported here. For instance, other interesting materials, such as $\mathrm{CsGeBr}_{3}$ [29] (used for second-harmonic generation in new infrared nonlinear system) or potassium hydrogen phthalate [30] (used in electro-optical modulators) belong to classes that are characterized by a 


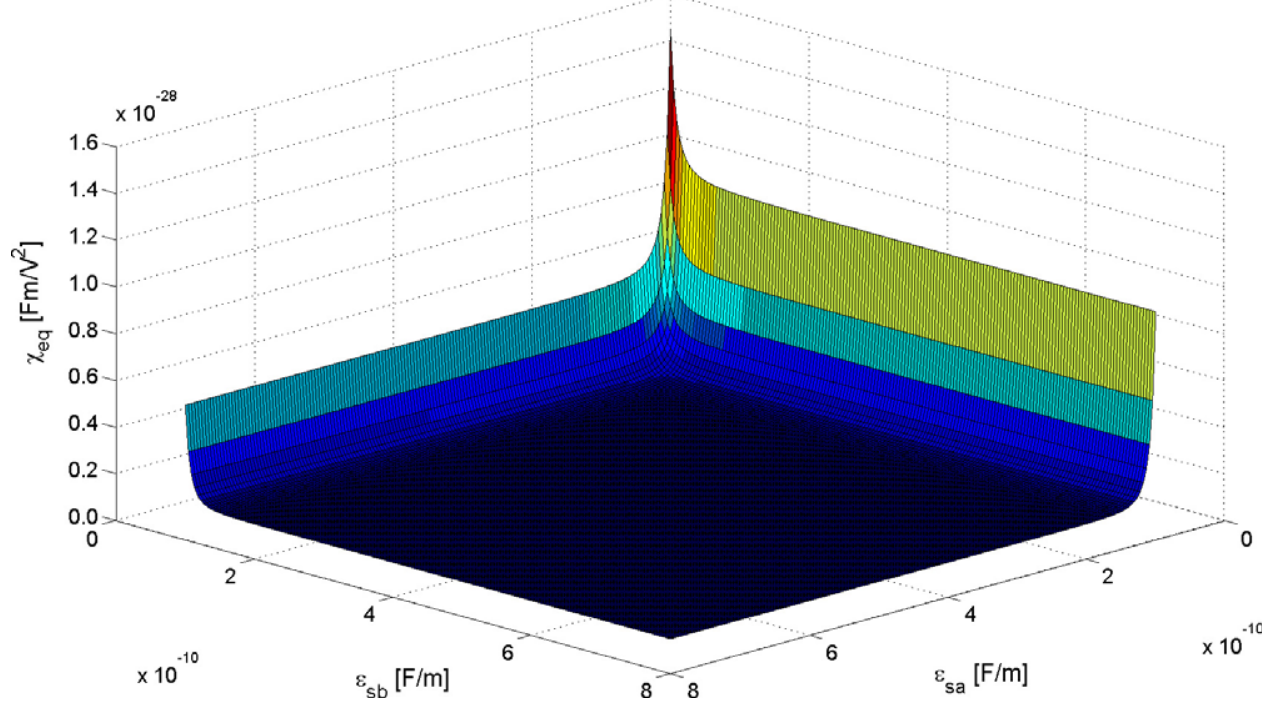

Figure 2. Equivalent third-order susceptibility versus $\varepsilon_{s a}$ and $\varepsilon_{s b}$ of inclusions belonging to the tetragonal-scalenohedral crystal class. Typical values of $\varepsilon_{0}=8.85 \times 10^{-12}\left(\mathrm{~F} \mathrm{~m}^{-1}\right), \chi_{s a}+\frac{1}{3} \chi_{s c}=$ $\chi_{s d}+\chi_{s e}=\chi_{s b}=2 \times 10^{-27}\left(\mathrm{~F} \mathrm{~m} \mathrm{~V}^{-2}\right), \eta_{s a}=\eta_{s b}=10^{-20}\left(\mathrm{~F} \mathrm{~V}^{-1}\right)$ and $c=0.1$ are used.

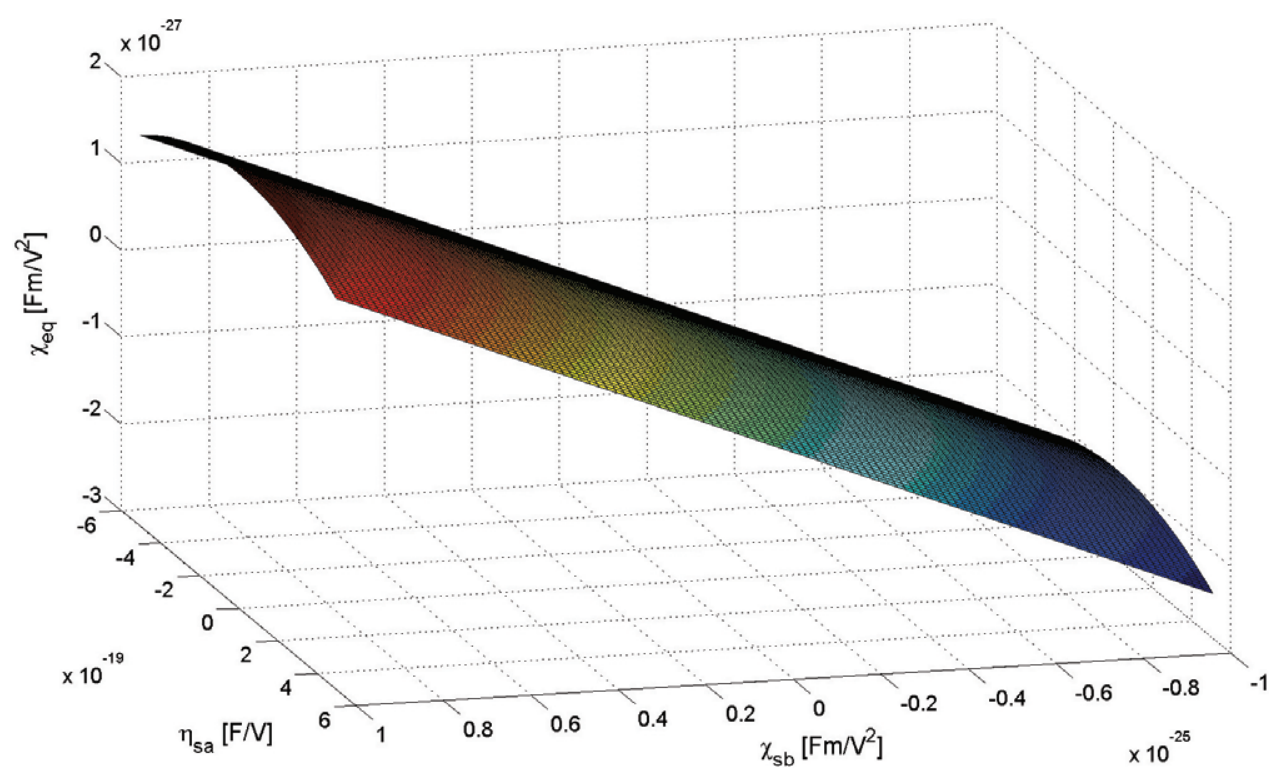

Figure 3. Equivalent susceptibility as a function of inclusion second- and third-order nonlinearities. Linear permittivity parameters of both matrix and inclusions are assumed to be equal to the vacuum dielectric constant, $8.85 \times 10^{-12}\left(\mathrm{~F} \mathrm{~m}^{-1}\right)$. In order to be able to assess the importance of the two terms, the following parameter assignment was made: $\chi_{s a}+\frac{1}{3} \chi_{s c}=\chi_{s d}+\chi_{s e}=0, \chi_{s b}$ ranging from $-1 \times 10^{-25}$ to $1 \times 10^{-25}\left(\mathrm{~F} \mathrm{~m} \mathrm{~V}^{-2}\right), \eta_{s b}=0$ and $\eta_{s a}$ ranging from $-5 \times 10^{-19}$ to $5 \times 10^{-19}\left(\mathrm{~F} \mathrm{~V}^{-1}\right)$

much larger number of free parameters. Explicit formulae would be quite cumbersome to write down here; however, they can be very well used in the modelling process. 


\section{Concluding remarks}

In this paper, we present a homogenizing procedure for a dispersion of anisotropic dielectrically nonlinear spheres embedded in a dielectrically linear host. The microstructure is such that the spherical grains are randomly oriented inside the hosting matrix. The overall material results in an isotropic nonlinear medium. Under the same hypothesis of the Maxwell-Garnett theory, we have found the electrical constitutive relation of the composite material as a function of the linear permittivity of the matrix and the three main tensors describing a single grain: $\varepsilon, \eta$ and $\chi$. The parameters describing the overall composite are the equivalent permittivity $\varepsilon_{\text {eq }}$ and the third-order scalar hypersusceptibility (the so-called Kerr equivalent susceptibility), $\chi_{\text {eq. }}$. An exact averaging procedure, performed over all the possible rotations of the spherical grains, has led to their explicit expressions in terms of the constitutive tensors. These parameters turn out to be two invariants of the system of the three tensors $\varepsilon, \eta$ and $\chi$. It is interesting to note that, whereas the single inclusion may have a quadratic nonlinearity, the macroscopic second-order term cancels out in the whole dispersion due to the macroscopic isotropy. Moreover, the third-order nonlinearity, which appears in the overall electrical behaviour of the composite material, depends also on the second-order term characterizing the inclusions.

Standard techniques pertaining to the field of nonlinear optics can be used to translate the results obtained in this work into the frequency domain [31]. The relationships obtained in this way require that the frequency behaviour of the materials is known and they hold valid provided the wavelengths coming into play are much larger than inclusion dimensions. Therefore, this methodology provides analytical expressions that can be proficiently used in the study and in the design of new composite materials with desired dielectric behaviour and optical response.

\section{Appendix A. Jahn convention and crystal classes}

In order to define the types of internal symmetry for tensors, we shall adopt a system of notation advocated by Jahn [27] and Sirotine [28]. We define the simple space of the functionals over vectors with the symbol $\mathrm{V}$. In this notation, $\mathrm{V}^{2}$ stands for the tensor product $\mathrm{V} \otimes \mathrm{V}$, $\left[\mathrm{V}^{2}\right]$ the space of symmetric second-order tensors, $\left[\mathrm{V}^{3}\right]$ the space of symmetric third-order tensors, $\mathrm{V}\left[\mathrm{V}^{2}\right]$ the tensor product of $\mathrm{V}$ and $\left[\mathrm{V}^{2}\right],\left[\left[\mathrm{V}^{2}\right]^{2}\right]$ the symmetric square of $\left[\mathrm{V}^{2}\right]$ (i.e., the symmetrized tensor product of $\left[\mathrm{V}^{2}\right]$ and $\left.\left[\mathrm{V}^{2}\right]\right), \mathrm{V}\left[\mathrm{V}^{3}\right]$ the tensor product of $\mathrm{V}$ and $\left[\mathrm{V}^{3}\right]$, and so on. Moreover, the skew-symmetry is indicated in a similar manner but adopting the curly braces $\{\cdot\}$. Thus, $\varepsilon$ belongs to $\left[\mathrm{V}^{2}\right], \eta$ belongs to $\mathrm{V}\left[\mathrm{V}^{2}\right]$ and $\chi$ belongs to $\mathrm{V}\left[\mathrm{V}^{3}\right]$. Due to Neumann's principle [25], any type of symmetry exhibited by the point group of a crystal is reflected in every physical property of the crystal. Therefore, in addition to properties given in equation (4), the constitutive tensors $\varepsilon, \eta$ and $\chi$ have other symmetries, according to the crystal class of the constituent material. When we are dealing with a given crystal class, each tensor belonging to a given Jahn type has a particular structure and a given number of independent entries, which is lower than the bound imposed by the internal symmetry. In table 1, we have reported, for all crystal classes, the actual number of independent entries of the tensors in the spaces $\left[\mathrm{V}^{2}\right], \mathrm{V}\left[\mathrm{V}^{2}\right]$ and $\mathrm{V}\left[\mathrm{V}^{3}\right]$, which can be of interest in the present work. For example, we may find in such a table that, in a ditetragonal-pyramidal crystal $(4 \mathrm{~mm})$, a tensor of the space $\left[\mathrm{V}^{2}\right]$ has two independent entries, a tensor in $\mathrm{V}\left[\mathrm{V}^{2}\right]$ has three independent entries and a tensor in $\mathrm{V}\left[\mathrm{V}^{3}\right]$ has five independent entries. In various textbooks [25], one can find the structure of many types of symmetric and skew-symmetric tensors for all the 32 crystal classes. 


\section{Appendix B. Electric field inside a nonlinear anisotropic sphere}

The symmetry hypothesis for the linear permittivity tensor $\varepsilon_{i j}^{0}$ ensures that it can be diagonalized with real eigenvalues. Therefore, a suitable basis exists, where equation (3) takes the following form:

$$
\varepsilon_{i j}\left(\mathbf{E}_{s}\right)=\varepsilon_{i} \delta_{i j}+\eta_{i j k} E_{k}+\chi_{i j k h} E_{k} E_{h} .
$$

From equation (B.1) on, tensors $\eta$ and $\chi$, as well as $\mathbf{E}_{0}$ and $\mathbf{E}_{s}$ are expressed in the basis where $\varepsilon^{0}$ is diagonal. Our aim is to find the explicit dependence of $\mathbf{E}_{s}$ on $\mathbf{E}_{0}$, which is valid when the nonlinear permittivity tensor is given by equation (B.1). From equations (2) and (B.1), we derive the following system of equations in the three components of $\mathbf{E}_{s}$ :

$$
\varepsilon_{j} E_{j} \delta_{i j}+\eta_{i j k} E_{j} E_{k}+\chi_{i j k h} E_{j} E_{k} E_{h}+2 \varepsilon_{0} E_{i}=3 \varepsilon_{0} E_{0 i} .
$$

In order to obtain a solution of the system (B.2) as a truncated series expansion in $\mathbf{E}_{0}$, we use the position $E_{i}=a_{j} E_{0 j} \delta_{i j}+b_{i j k} E_{0 j} E_{0 k}+c_{i j k h} E_{0 j} E_{0 k} E_{0 h}$. By substituting this form of the solution in equation (B.2) and by separating the terms of the same order, we identify the coefficient matrices $a, b$ and $c$. This leads to the following expressions:

$$
\begin{aligned}
a_{j} & =\frac{3 \varepsilon_{0}}{\varepsilon_{j}+2 \varepsilon_{0}}, \\
b_{i j k} & =-\frac{a_{i} a_{j} a_{k} \eta_{i j k}}{3 \varepsilon_{0}}, \\
c_{i j k h} & =\frac{a_{i} a_{j} a_{k} a_{h}}{9 \varepsilon_{0}^{2}}\left(2 \sum_{q} a_{q} \eta_{i j q} \eta_{q k h}-3 \varepsilon_{0} \chi_{i j k h}\right) .
\end{aligned}
$$

(In equations (B.4) and (B.5), repeated indices do not indicate implicit sums. In equation (B.5) the sole summed index is $q$.) Moreover, we note that, in order to get to equation (B.5) we had to use the symmetry property $\eta_{i j k}=\eta_{i k j}$.

\section{Appendix C. Averaging higher-order unit vector products}

We shall use Euler angle representation $(\psi, \varphi$ and $\vartheta)$ to write down the explicit expressions for the components of the unit vectors $\mathbf{n}_{1}, \mathbf{n}_{2}$ and $\mathbf{n}_{3}$ :

$\mathbf{n}_{1}=(\cos \psi \cos \varphi-\sin \psi \sin \varphi \cos \theta,-\cos \psi \sin \varphi-\sin \psi \cos \varphi \cos \theta, \sin \psi \sin \theta)$

$\mathbf{n}_{2}=(\sin \psi \cos \varphi+\cos \psi \sin \varphi \cos \theta,-\sin \psi \sin \varphi+\cos \psi \cos \varphi \cos \theta,-\cos \psi \sin \theta)$

$\mathbf{n}_{3}=(\sin \varphi \sin \theta, \sin \theta \cos \varphi, \cos \theta)$.

In order to obtain $\left\langle\mathbf{E}_{s}\right\rangle$, we need to calculate the average value of the quantities $n_{i l} n_{i r}, n_{j l} n_{k s} n_{i r}$ and $n_{j l} n_{k s} n_{h t} n_{i r}$. This is done by the following integrals:

$$
\begin{aligned}
& \left\langle n_{i l} n_{i r}\right\rangle=\frac{1}{8 \pi^{2}} \int_{0}^{2 \pi} \int_{0}^{2 \pi} \int_{0}^{\pi} n_{i l} n_{i r} \sin \theta \mathrm{d} \theta \mathrm{d} \varphi \mathrm{d} \Psi \\
& \left\langle n_{j l} n_{k s} n_{i r}\right\rangle=\frac{1}{8 \pi^{2}} \int_{0}^{2 \pi} \int_{0}^{2 \pi} \int_{0}^{\pi} n_{j l} n_{k s} n_{i r} \sin \theta \mathrm{d} \theta \mathrm{d} \varphi \mathrm{d} \Psi \\
& \left\langle n_{j l} n_{k s} n_{h t} n_{i r}\right\rangle=\frac{1}{8 \pi^{2}} \int_{0}^{2 \pi} \int_{0}^{2 \pi} \int_{0}^{\pi} n_{j l} n_{k s} n_{h t} n_{i r} \sin \theta \mathrm{d} \theta \mathrm{d} \varphi \mathrm{d} \Psi .
\end{aligned}
$$

After some straightforward computations, we solve the first equation:

$$
\left\langle n_{i l} n_{i r}\right\rangle=\frac{1}{3} \delta_{l r} .
$$


More work is needed to obtain the second average. It turns out that it is different from zero only if $\{j k i\}$ and $\{l s r\}$ are permutations of the basic set $\{123\}$. Moreover, the result is $1 / 6$ if the permutation that maps $\{j k i\}$ to $\{l s r\}$ is even and it is $-1 / 6$ if this permutation is odd; we synthesize this by means of the generalized Kronecker delta:

$$
\left\langle n_{j l} n_{k s} n_{i r}\right\rangle=\frac{1}{6} \delta_{j k i}^{l s r} \text {. }
$$

We recall that the generalized Kronecker delta, denoted by $\delta_{j k i}^{l s r}$, is zero if there is a repetition in the sets $\{j k i\}$ or $\{l s r\}$ or if $\{j k i\} \neq\{l s r\}$ as sets. If none of the above conditions are met, then $\delta_{j k i}^{l s}$ is defined as the sign of the permutation that maps $\{j k i\}$ to $\{l s r\}$.

The determination of the average value of $n_{j l} n_{k s} n_{h t} n_{i r}$ leads to four main cases. When $j=k=h=i$ we are dealing with a single unit vector, say $\hat{\mathbf{n}}=\left(\hat{n}_{1}, \hat{n}_{2}, \hat{n}_{3}\right)$, and a long but straightforward integration leads to the result:

$$
\left\langle\hat{n}_{l} \hat{n}_{s} \hat{n}_{t} \hat{n}_{r}\right\rangle=\frac{1}{15}\left(\delta_{l s} \delta_{t r}+\delta_{l t} \delta_{s r}+\delta_{l r} \delta_{s t}\right) \triangleq[l s t r] .
$$

The second case deals with the situation with two different index pairs in the set $\{j k h i\}$. So, we consider two orthogonal unit vectors, say $\hat{\mathbf{n}}$ and $\hat{\mathbf{m}}=\left(\hat{m}_{1}, \hat{m}_{2}, \hat{m}_{3}\right)$. As before, the integration provides

$$
\left\langle\hat{n}_{l} \hat{n}_{s} \hat{m}_{t} \hat{m}_{r}\right\rangle=\frac{2}{15} \delta_{l s} \delta_{t r}-\frac{1}{30}\left(\delta_{l t} \delta_{s r}+\delta_{l r} \delta_{s t}\right) \triangleq\{l s, t r\} .
$$

The third case deals with the situation where the three indices in the set $\{j k h i\}$ are equal and the remainder is different from them. As before, we consider two orthogonal unit vectors, say $\hat{\mathbf{n}}$ and $\hat{\mathbf{m}}$. Now, the integration always provides the result zero:

$$
\left\langle\hat{n}_{l} \hat{n}_{s} \hat{n}_{t} \hat{m}_{r}\right\rangle=0 .
$$

Finally, the fourth case deals with the situation with two equal indices in the set $\{j k h i\}$ and two indices different from them. So, we consider three orthogonal unit vectors, say $\hat{\mathbf{n}}, \hat{\mathbf{m}}$ and $\hat{\mathbf{u}}=\left(\hat{u}_{1}, \hat{u}_{2}, \hat{u}_{3}\right)$. As before, the integration always provides the result zero:

$$
\left\langle\hat{n}_{l} \hat{n}_{s} \hat{m}_{t} \hat{u}_{r}\right\rangle=0 \text {. }
$$

The results shown in equations (C.5)-(C.8) may be used to obtain the requested average value of the quantity $n_{j l} n_{k s} n_{h t} n_{i r}$. Eventually, we may write the final formula:

$$
\begin{aligned}
\left\langle n_{j l} n_{k s} n_{h t} n_{i r}\right\rangle & =\delta_{j k} \delta_{k h} \delta_{h i}[l s t r]+\delta_{j k} \delta_{h i}\left(1-\delta_{k h}\right)\{l s, t r\} \\
& +\delta_{j h} \delta_{k i}\left(1-\delta_{k h}\right)\{l t, s r\}+\delta_{j i} \delta_{k h}\left(1-\delta_{k i}\right)\{l r, s t\} .
\end{aligned}
$$

By substituting the averaging symbols defined in equations (C.5) and (C.6), we obtain the following form of this result:

$$
\begin{aligned}
\left\langle n_{j l} n_{k s} n_{h t} n_{i r}\right\rangle & =\frac{2}{15} \delta_{l s} \delta_{t r}\left[\delta_{j k} \delta_{h i}-\frac{1}{4}\left(\delta_{j h} \delta_{k i}+\delta_{j i} \delta_{k h}\right)\right] \\
& +\frac{2}{15} \delta_{l t} \delta_{s r}\left[\delta_{j h} \delta_{k i}-\frac{1}{4}\left(\delta_{j k} \delta_{h i}+\delta_{j i} \delta_{k h}\right)\right] \\
& +\frac{2}{15} \delta_{l r} \delta_{s t}\left[\delta_{j i} \delta_{k h}-\frac{1}{4}\left(\delta_{j h} \delta_{k i}+\delta_{j k} \delta_{h i}\right)\right]
\end{aligned}
$$

\section{Appendix D. Invariance of the macroscopic parameters with respect to the reference frame}

In order to show that the equivalent permittivity $\varepsilon_{\text {eq }}$ is an invariant, it is sufficient to write the parameter $s$ that appears in equation (19) in terms of the classical invariants of the linear permittivity tensor:

$$
s=\frac{3 I_{3}+3 \varepsilon_{0} I_{2}-12 \varepsilon_{0}^{3}}{I_{3}+2 \varepsilon_{0} I_{2}+4 \varepsilon_{0}^{2} I_{1}+8 \varepsilon_{0}^{3}},
$$


where the three invariants are defined as follows:

$$
\begin{aligned}
& I_{1}=\operatorname{tr} \varepsilon \\
& I_{2}=\frac{1}{2}\left[(\operatorname{tr} \varepsilon)^{2}-\operatorname{tr}\left(\varepsilon^{2}\right)\right] \\
& I_{3}=\operatorname{det} \varepsilon .
\end{aligned}
$$

Similarly, one can verify that the equivalent hypersusceptibility $\chi_{\text {eq }}$ is an invariant quantity depending only on the tensors $\varepsilon, \eta$ and $\chi$. This is true since $\chi_{\mathrm{eq}}$ can be written as subsequent products and contractions performed over them. More precisely, we may observe that the parameters $\alpha$ and $\beta$, which appear inside the expression for $\chi_{\text {eq }}$, can be written in a tensorial form that explicitly unveils their invariant character. If we introduce the tensor $\gamma=(\varepsilon+2 I)^{-1}$, we obtain the following relationships:

$\alpha=\varepsilon_{0} \operatorname{tr}(\gamma)$,

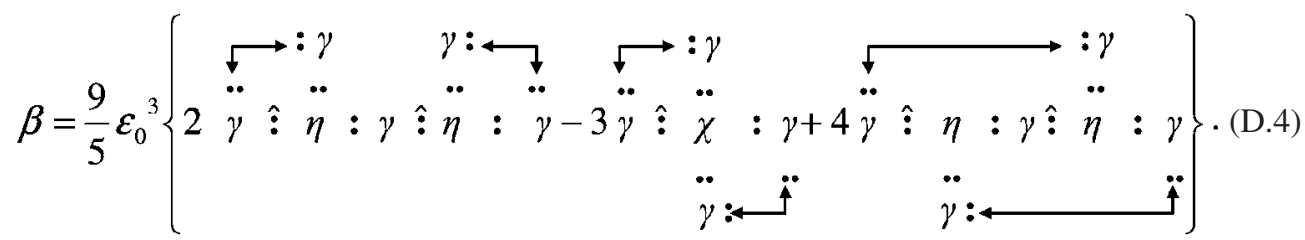

In these formulae, 'tr' means trace of the tensor, the symbol ' $:$ ' represents the saturation of two indices in the adjacent tensors and the symbol ' $\hat{:}$ ' represents a saturation between the first index in the tensor $\eta$ or $\chi$ and the adjacent tensor (we recall that the first index, in such tensors, has a behaviour different from the others because it is not involved in the internal symmetries). Equation (D.4) may seem complicated but it has a simple meaning. The output parameter $\beta$ is scalar and therefore all the indices involved in its definition must be completely saturated. In this context, the number of indices of a tensor is similar to the number of chemical bonds that an atom can support. Therefore, equation (D.4) can be seen as a chemical formula of a molecule where all bonds are indicated by means of the symbol ' $:$ '. Because of the symmetries, all the bonds are equivalent except for those involving the first index of the tensors $\eta$ or $\chi$, which are indicated with ' $\hat{\imath}$ '. As one can see, the previous expressions are the tensorial counterpart of equation (9) that defines the parameters $\alpha$ and $\beta$ and are written only in terms of products and contractions of tensors.

Finally, it is important to remember that equation (9) or, equivalently, equations (D.3) and (D.4), providing the most important parameters of this theory, are correct only if the constitutive tensors of the system fulfil the symmetry conditions established in equation (4). If the tensors $\eta$ and $\chi$ are known in a different, asymmetric form $\tilde{\eta}$ and $\tilde{\chi}$, one can perform some standard symmetrization in order to apply the present theory:

$$
\begin{aligned}
& \eta_{i j k}=\frac{1}{2} \sum_{(j k) \in \sigma_{2}} \tilde{\eta}_{i(j k)} \\
& \chi_{i j k h}=\frac{1}{6} \sum_{(j k h) \in \sigma_{3}} \tilde{\chi}_{i(j k h)} .
\end{aligned}
$$

The summations extend over all permutations $\sigma_{n}$ (over $n$ elements) of the indices inside the brackets. As it is known, symmetrization is a tensor operation.

\section{References}

[1] Maxwell J C 1881 A Treatise on Electricity and Magnetism (Oxford: Clarendon)

[2] Maxwell-Garnett J C 1904 Phil. Trans. R. Soc. B 203385

[3] Bruggeman D A G 1935 Ann. Phys., Lpz. 24636 
[4] Van Beek L H K 1967 Prog. Dielectr. 771

[5] Giordano S 2003 J. Electrost. 5859

[6] Duan H L, Karihloo B L, Wang J and Yi X 2006 Phys. Rev. B 73174203

[7] Hashin Z and Shtrikman S 1962 J. Appl. Phys. 333125

[8] Brown W F 1955 J. Chem. Phys. 231514

[9] Yu K W, Hui P M and Stroud D 1993 Phys. Rev. B 4714150

Yu K W, Hui P M and Stroud D 1997 Phys. Rev. B 5614195 (erratum)

[10] Zhang X and Stroud D 1994 Phys. Rev. B 49944

[11] Stroud D 1996 Phys. Rev. B 543295

[12] Barabash S and Stroud D 1999 J. Phys.: Condens. Matter 1110323

[13] Liu B, Gao L and Yu K W 2005 Phys. Rev. B 72214208

[14] Wei E B and Wu Z K 2004 J. Phys.: Condens. Matter 16 5377-86

[15] Lakhtakia M N and Lakhtakia A 2001 Electromagnetics 21 129-38

[16] Mackay T G, Lakhtakia A and Weiglhofer W S 2002 Opt. Commun. 204 219-28

[17] Giordano S and Rocchia W 2005 J. Appl. Phys. 98104101

[18] Torquato S and Sen A K 1990 J. Appl. Phys. 671145

[19] Levy O and Stroud D 1997 Phys. Rev. B 568035

[20] Nagatani T 1980 J. Appl. Phys. 514944

[21] Giordano S 2006 J. Electrost. 64655

[22] Hui P M and Stroud D 1997 J. Appl. Phys. 824740

[23] Hui P M, Cheung P and Stroud D 1998 J. Appl. Phys. 843451

[24] Cherepanov A and Sihvola A 1996 J. Electromagn. Waves Appl. 1079

[25] Sirotine Y and Chaskolskaia M 1984 Fondaments de la Physique des Cristaux (Moscow: Editions MIR)

[26] Dmitriev V and Tarassov L 1987 Optique Non Linéaire Appliquée (Moscow: Editions MIR)

[27] Jahn H A 1949 Acta Crystallogr. 230

[28] Sirotine Y 1975 Sov. Phys.-Crystallogr. 19565

[29] Tang L C, Huang J Y, Chang C S, Lee M H and Liu L Q 2005 J. Phys.: Condens. Matter 17 7275-86

[30] Kejalakshmy N and Srinivasan K 2003 J. Phys. D: Appl. Phys. 36 1778-82

[31] Shen Y R 2003 The Principles of Nonlinear Optics (Hoboken: Wiley) 